\title{
Does cartilage loss cause pain in osteoarthritis and if so, how much?
}

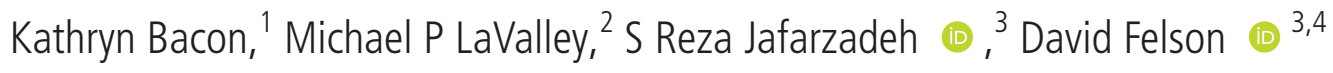

\section{Handling editor Josef S}

Smolen

${ }^{1}$ Rheumatology, Boston

University, Boston,

Massachusetts, USA

${ }^{2}$ Biostatistics, School of Public Health, Boston, Massachusetts, USA

${ }^{3}$ Section of Rheumatology, Boston University School of Medicine, Boston,

Massachusetts, USA

${ }^{4}$ Centre for Epidemiology Versus Arthritis, Centre for Musculoskeletal Research, The University of Manchester and NIHR Manchester Musculoskeletal Biomedical Research Centre, Manchester University NHS Foundation Trust, Manchester Academic Health Science Centre, Manchester, UK

Correspondence to

Professor David Felson, boston university, Boston,

Massachusetts, USA dfelson@bu.edu

Received 18 March 2020

Revised 18 April 2020

Accepted 21 April 2020

Published Online First

7 May 2020

\section{ABSTRACT \\ Objectives Although treatment development in} osteoarthritis $(\mathrm{OA})$ focuses on chondroprotection, it is unclear how much preventing cartilage loss reduces joint pain. It is also unclear how nociceptive tissues may be involved.

Methods Using data from the Osteoarthritis Initiative, we quantified the relation between cartilage loss and worsening knee pain after adjusting for bone marrow lesions (BMLs) and synovitis, and examined how much these factors mediated this association. 600 knee MRIs were scored at baseline, 12 months and 24 months for quantitative and semiquantitative measures of $\mathrm{OA}$ structural features. We focused on change in medial cartilage thickness using an amount similar to that seen in recent trials. Linear models calculated mean change in Western Ontario and McMaster Universities Osteoarthritis Index (WOMAC) pain score with cartilage loss, adjusted for baseline BMLs, synovitis and covariates. Mediation analysis tested whether change in synovitis or BMLs mediated the cartilage loss-pain association. We carried out a subanalysis for knees with non-zero baseline WOMAC pain scores and another for non-valgus knees.

Results Cartilage thickness loss was significantly associated with a small degree of worsening in pain over 24 months. For example, a loss of $0.1 \mathrm{~mm}$ of cartilage thickness over 2 years was associated with a 0.32 increase in WOMAC pain (scale 0-20). The association of cartilage thickness loss with pain was mediated by synovitis change but not by BML change. Subanalysis results were similar.

Conclusions Cartilage thickness loss is associated with only a small amount of worsening knee pain, an association mediated in part by worsening synovitis. Demonstrating that chondroprotection reduces knee pain will be extremely challenging and is perhaps unachievable.

Osteoarthritis (OA) is the most common form of arthritis and one of the leading causes of disability worldwide. ${ }^{1}$ There are no approved treatments demonstrated to slow disease progression and for many years treatment development has focused on protection against cartilage loss. The main symptom associated with OA is pain and the relation between chondroprotection and pain relief has not been clear.

Intact cartilage is not innervated with pain fibres, although with disease, small pores at the osteochondral junction bring neurovascular inputs into deep layers of cartilage and could provide a source of pain. ${ }^{2}$ Furthermore, chondrocytes synthesise

\section{Key messages}

What is already known about this subject?

- The goal of much treatment development in osteoarthritis has been to identify a treatment that both protects against cartilage loss and relieves joint pain.

What does this study add?

- Using data from the Osteoarthritis Initiative which followed participants over 36 months with annual MRIs and pain surveys, we found that cartilage loss was associated with only a tiny amount of worsening knee pain. Some of this association was mediated by worsening synovitis.

How might this impact on clinical practice or future developments?

- Cartilage loss has such a tiny effect on pain that demonstrating that a chondroprotective treatment reduces joint pain may not be achievable.

nerve growth factor, a potential source of pain. ${ }^{3}$ Thus, although other articular tissues such as the synovium and bone are richly innervated with nerve fibres and are thought to be sources of pain, cartilage loss could trigger pain directly or indirectly when proteolytic fragments of different cartilage matrix molecules activate Toll-like receptors that act as damage associated molecular patterns (DAMPs). ${ }^{245}$

Studies examining the relation of cartilage loss with pain initially focused on joint space loss on the $\mathrm{X}$-ray, a proxy for cartilage loss. In general, these studies found little relation of joint space loss with worsening pain. ${ }^{6-10}$ Cross-sectional studies with MRI data showed that persons with less cartilage tended to have more pain, ${ }^{11} 12$ but few longitudinal studies have examined cartilage loss over time and its correlation with change in knee pain. A small Australian study by Wluka and colleagues ${ }^{6}$ showed a weak but statistically significant association of cartilage volume loss in the tibia with worsening pain over a 2-3-year period; Eckstein and associates, ${ }^{13}$ using data from the Osteoarthritis Initiative (OAI) cohort, also found that, compared with those who experienced no worsening pain, participants with pain worsening had a slightly higher odds of cartilage loss over 24 months.

In a recently published randomised placebocontrolled trial, ${ }^{14}$ Hochberg and colleagues reported that sprifermin, an agonist of fibroblast 
growth factor (FGF)-18, prevented cartilage loss in the knee, but had no effect on knee pain. These trial results have once again raised concerns that the relation of cartilage loss with worsening pain is so weak that treatments that abrogate loss might not have a detectable effect on pain. If so, no treatment targeting cartilage protection would be likely to succeed as a Disease Modifying Osteoarthritis Drug.

There is an indirect path by which cartilage loss could affect pain. The relationship could be through structures within the knee now widely thought to cause pain because of their rich nociceptive innervation, the synovium and bone marrow. ${ }^{245}$ Since cartilage loss co-occurs with synovitis and bone marrow lesions (BMLs) in OA, in cross-sectional analyses the latter two lesions could confound an association of cartilage loss with pain. In longitudinal studies, an effect of cartilage loss on pain could be due to the intermediate effect of cartilage loss promoting synovitis or BMLs which then cause pain. Such a relationship would be described as mediation.

Using observational data from the Foundation for the National Institutes of Health (FNIH) cohort within the OAI, ${ }^{13} 15$ we quantified the relation of cartilage loss and worsening knee pain after adjusting for BMLs and synovitis at baseline and then examined whether the association of cartilage loss with worsening pain was mediated by worsening synovitis or change in BMLs.

\section{PATIENTS AND METHODS Study population}

Data for these analyses are from the public use data set(s) of the OAI, including data from the FNIH OA Biomarkers Consortium Project $^{16}$ (available at https://nda.nih.gov/oai). The OAI included men and women ages 45-79 with or at risk for symptomatic knee OA. Our study sample included 600 subjects who were selected for a case- control study nested within the OAI. There were four subgroups within the 600 subjects. One subgroup of knees $(n=194)$ had both medial tibiofemoral radiographic joint space loss $(\geq 0.7 \mathrm{~mm})$ and a persistent increase in the Western Ontario and McMaster Universities Osteoarthritis Index (WOMAC) pain score ( $\geq 9$ on a $0-100$ scale) $24-48$ months from baseline. Other subgroups included 200 subjects with neither radiographic nor pain progression, 103 with radiographic progression only and 103 with pain progression only. All subject knees underwent 3T knee MRI both at baseline and follow-up. We used the score from one knee MRI per subject at the baseline, 12-month, and 24-month OAI visits using quantitative assessments of cartilage thickness and volume, ${ }^{17} 18$ and semiquantitative assessments of structural features of OA using the MRI Osteoarthritis Knee Score (MOAKS). ${ }^{19}$ Figure 1 shows a time line on assessment of key variables.

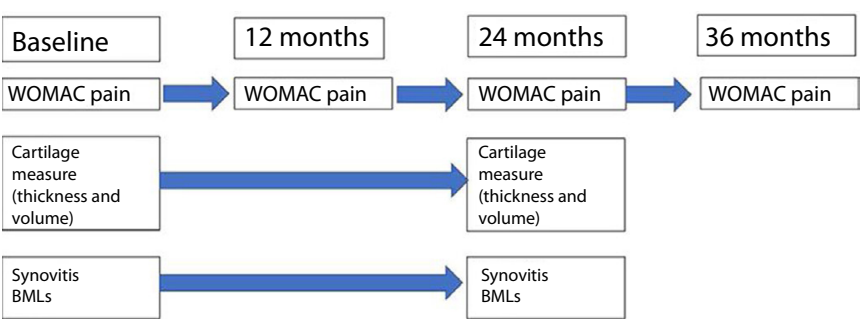

Figure 1 Timeline for key measures. BMLs, bonemarrow lesions; WOMAC, WesternOntario and McMaster Universities Osteoarthritis Index.

\section{Cartilage measures}

For our primary analysis, we focused on a quantitative measure of cartilage in the medial joint: mean cartilage thickness over the total area of subchondral bone $(\mathrm{mm}) .{ }^{17}{ }^{18}$ Change in cartilage thickness loss was calculated as:

(later visit score) - (baseline visit score)

We calculated change from baseline to 24 months. The cartilage change score was then multiplied by -1 so that in the case of a reduction of cartilage, the worsening of cartilage loss would have a positive value. We scaled this value as 0.1 and $0.05 \mathrm{~mm}$ because this amount of change has been seen in prior studies. For example, in the recent Sprifermin study, ${ }^{14}$ in the highest dose, cartilage thickness loss of $0.05 \mathrm{~mm}$ per year was prevented which translates into $0.1 \mathrm{~mm}$ over 2 years. An earlier trial reported $0.1 \mathrm{~mm}$ medial cartilage thickness loss in controls over 2 years and reported that steroids would induce loss of cartilage thickness by an additional $0.10 \mathrm{~mm}$ over 2 years. ${ }^{20}$ We tested the possibility of chondroprotective treatment that would be very effective, preventing all cartilage loss over that period and also tested one that would prevent half the loss $(0.05 \mathrm{~mm})$.

In a secondary analysis, we used semiquantitative measures of cartilage loss from the MOAKS scores on the MRIs. ${ }^{19}$ At each visit, we created summary scores for cartilage thickness loss and cartilage area loss by summing 14 region scores (each region score range 0-3) for each measure. We then calculated change from baseline to 24 months for both summary MOAKS cartilage loss scores.

\section{Knee pain}

Knee pain was assessed at each visit using the WOMAC pain subscale. ${ }^{21}{ }^{22}$ We used the WOMAC knee pain score (range 0-20) from the 'MRI knee' from each visit and calculated change from baseline to 24 and 36 months. This allowed us to evaluate the association of the cartilage loss exposure with pain over shorter and longer periods, while the change in pain to 36 months value also established an outcome occurring after the evaluation of the other key variables.

\section{Bone marrow lesions}

For each visit, we created a summary score for BMLs (range $0-45$, sum of 15 region BML size scores) and computed change scores from baseline to 24 months. ${ }^{19}$

\section{Synovitis}

For each visit, we created a synovitis summary score (range $0-6)$ using the sum of Hoffa-synovitis and effusion scores. ${ }^{19}$ We computed change scores from baseline to 24 months.

\section{Statistical methods}

Linear models were used to calculate the response mean change in WOMAC pain associated with cartilage loss, adjusted for baseline WOMAC pain, BMLs, age, sex, body mass index (BMI) $\left(\mathrm{kg} / \mathrm{m}^{2}\right)$, race (caucasian, African-American, other non-caucasian) and baseline depressive symptoms using a score of $\geq 16$ from the Center for Epidemiologic Studies Depression Scale. ${ }^{23}$

We used mediation analysis ${ }^{24} 25$ to examine a model of describing how the total effect of cartilage loss on pain change to 24 or 36 months might be broken down into the 'controlled direct effect' of cartilage loss on pain, and the 'indirect effect' on a pathway through synovitis or BML change (figure 2). We calculated the mediation proportion ${ }^{25-27}$ and its $95 \%$ CI to estimate the proportion of the cartilage loss to pain effect attributable to 


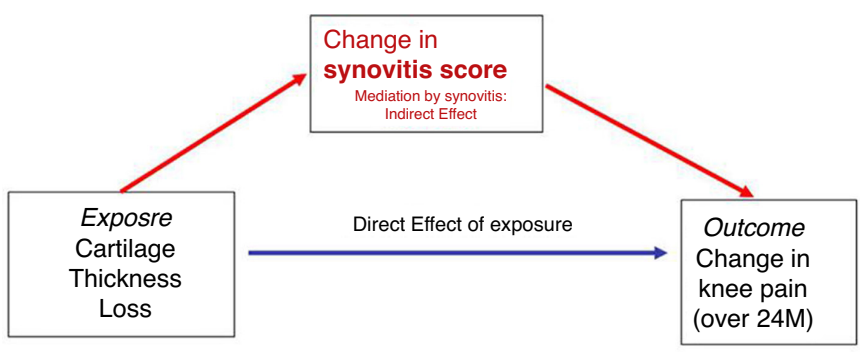

Total Effect $=$ Direct Effect + Indirect Effect

Figure 2 DAG for mediation relationships: DAG of proposed relationship between exposure cartilage thickness loss over 24 months, and outcome change in WOMAC knee pain over 24 months, with mediation by change in synovitis over 24 months. Confounders used in the analysis are not shown for simplicity. Mediation by change in BMLs: a similar model is proposed for mediation by change in BMLs. BMLs, bonemarrow lesions; DAG, directed acyclic graph; WOMAC, Western Ontario and McMaster Universities Osteoarthritis Index.

the pathway including the mediator. Mediation was estimated separately for change in synovitis and change in BMLs.

To interpret the direct and indirect effects estimated in a mediation analysis, we addressed three assumptions ${ }^{28}$ related to confounding: confounders for the exposure-outcome relationship (in this case, the relation of cartilage loss to change in pain), the exposure-mediator relationship (relation of cartilage loss to change in synovitis or change in BMLs) and the mediator-outcome relationship (relation of either change in synovitis or change in BMLs to the outcome change in pain) must all be adjusted for in the analysis. We have adjusted for a set of covariates that should address these requirements. In addition, a fourth assumption is required that there should be no mediator-outcome confounder which is affected by the exposure; we asserted that this should also hold.

We carried out several subanalyses. The first subanalysis was limited to knees with non-zero WOMAC pain scores at baseline, as those would likely be treated. The second subanalysis limited the sample to knees with baseline WOMAC pain score of 0 . The third subanalysis excluded knees with valgus alignment, as most knee OA is medial, and we used medial cartilage measures. Knees with valgus alignment and OA might have more lateral cartilage loss than medial cartilage loss.

Neither patients nor public were involved in this analysis of deidentified data from a completed study.

\section{RESULTS}

The characteristics of the study sample are shown in table 1. The sample consisted mostly of women (59\%), of mean age 62 years $(\mathrm{SD}=8.9)$ with mean BMI $31 \mathrm{~kg} / \mathrm{m}^{2}(\mathrm{SD}=4.8)$. Prevalence of depressive symptoms was $10 \%$. There was a predominance of individuals with mid-range OA by K\&L grade, rather than no or mild disease. While the baseline WOMAC pain score averaged 2.4 , it was 4.0 in those with non-zero WOMAC pain. The mean WOMAC score increased slightly over 24 or 36 months $(0.62$ and 0.93 , respectively, on a $0-20$ scale). Cartilage thinned in the medial tibiofemoral compartment over 24 months $(-0.10 \mathrm{~mm})$. Synovitis score increased (worsened) over 24 months (0.23), while the BML size summary score decreased slightly over 24 months $(-0.45)$.
Table 1 Study sample characteristics: baseline values and change over follow-up $(n=600)$

\begin{tabular}{|c|c|}
\hline & Mean (SD) \\
\hline Age & $62(8.9)$ \\
\hline Female (\%) & $59 \%$ \\
\hline African-American (\%) & $18 \%$ \\
\hline BMI $\left(\mathrm{kg} / \mathrm{m}^{2}\right)$ & $31(4.8)^{*}$ \\
\hline Depressive symptoms (CESD) & $10 \% \dagger$ \\
\hline \multicolumn{2}{|l|}{ Kellgren and Lawrence Grade } \\
\hline 0 & $0 \%$ \\
\hline 1 & $12.5 \%$ \\
\hline 2 & $51.0 \%$ \\
\hline 3 & $36.5 \%$ \\
\hline WOMAC pain score baseline (range 0 to 20) & $2.4(3.1)$ \\
\hline Change, baseline to 24 months & $0.62(3.4)$ \\
\hline Change, baseline to 36 months & $0.93(3.7) \dagger$ \\
\hline \multicolumn{2}{|c|}{ Cartilage thickness $(\mathrm{mm})$, medial tibiofemoral compartment } \\
\hline Baseline (range 1 to 8.6) & $3.3(0.61)$ \\
\hline Change, baseline to 24 months (range -2 to 2 ) & $-0.10(0.21)^{*}$ \\
\hline \multicolumn{2}{|l|}{ Cartilage thickness score (MOAKS) } \\
\hline Baseline score (range 0 to 42) & $2.8(2.8)$ \\
\hline Change, baseline to 24 months (range \pm 42 ) & $0.79(1.56)$ \\
\hline \multicolumn{2}{|l|}{ Synovitis score (MOAKS) } \\
\hline Baseline (range 0-6) & $1.53(1.19)$ \\
\hline Change, baseline to 24 months (range \pm 6 ) & $0.23(0.88)$ \\
\hline \multicolumn{2}{|l|}{ BML size summary score (MOAKS) } \\
\hline Baseline (range 0 to 45) & $3.78(2.79)$ \\
\hline Change, baseline to 24 months (range \pm 45 ) & $-0.45(2.34)^{*}$ \\
\hline \multicolumn{2}{|c|}{$\begin{array}{l}\text { Values are mean(SD) unless otherwise specified. } \\
{ }^{*} \mathrm{n}=599 . \\
\mathrm{tn}=596 . \\
\text { BMI, body mass index; BML, bone marrow lesion; CESD, Center for Epidemiologic } \\
\text { Studies Depression Scale; MOAKS, MRI Osteoarthritis Knee Score; WOMAC, Western } \\
\text { Ontario and McMaster Universities Osteoarthritis Index. }\end{array}$} \\
\hline
\end{tabular}

Cartilage thickness loss was significantly associated with a small degree of worsening pain over 24 or 36 months (table 2). On the 0-20 WOMAC pain scale, cartilage loss of $0.1 \mathrm{~mm}$ over 24 months translated to a 0.32 higher WOMAC pain score (95\% CI 0.21, 0.44) (scale 0-20). The effect of cartilage thickness loss over 24 months on pain change over 36 months was weaker but still statistically significant $(0.19$ higher WOMAC pain score for cartilage loss of $0.10 \mathrm{~m}$ over 24 months $(95 \% \mathrm{CI}$ $0.07,0.32))$.

Cartilage thickness loss was significantly associated with increasing synovitis score $(\mathrm{p}<0.0001)$ and its association with increasing BML score was statistically borderline $(\mathrm{p}=0.08)$. The association of cartilage thickness loss with increased WOMAC pain over 24 months was substantially mediated by the synovitis score change, but much less so by BML score change (table 3) (mediation proportion for synovitis change was 14\% for cartilage thickness loss). We found similar results for cartilage thickness loss over 24 months and pain from baseline to 36 months (table 4).

Subanalyses limited to those with non-zero WOMAC pain scores at baseline $(n=367)$ gave a significant association of cartilage thickness loss with pain that was very similar to that seen in the primary analysis sample (cartilage thickness loss of $0.1 \mathrm{~mm}$ over 24 months corresponded to an increase in WOMAC pain over 24 months of $0.33,95 \%$ CI $0.17,0.5)$. The subanalysis limited to those with no baseline pain $(n=233)$ also gave similar results to the primary analysis, although a $0.1 \mathrm{~mm}$ loss in 
Table 2 Association of cartilage thickness loss over 24 months with change in WOMAC knee pain over 24 or 36 months

\begin{tabular}{lll}
\hline & $\begin{array}{l}\text { Outcome over } 24 \text { months } \\
\text { Mean change in WOMAC knee pain over 24 months* } \\
(95 \% \mathrm{Cl})\end{array}$ & $\begin{array}{l}\text { Outcome over } 36 \text { months } \\
\text { Mean change in WOMAC knee pain over 36 months* } \\
(95 \% \mathrm{Cl})\end{array}$ \\
\hline $\begin{array}{l}\text { Exposure: } 0.1 \mathrm{~mm} \text { cartilage thickness loss over } 24 \\
\text { months }\end{array}$ & $\begin{array}{l}0.32(0.21 \text { to } 0.44) \dagger \\
\mathrm{p}<0.0001\end{array}$ & $\begin{array}{l}0.19(0.07 \text { to } 0.32) \dagger \\
\mathrm{p}=0.0025\end{array}$ \\
\hline BML at baseline (size summary score across 15 regions) & $0.10(0.00$ to 0.19$)$ & $0.18(0.08$ to 0.28$)$ \\
& $\mathrm{p}=0.04$ & $\mathrm{p}=0.0005$ \\
\hline Synovitis at baseline (summary score) & $0.03(-0.18$ to 0.24$)$ & $-0.05(-0.28$ to 0.18$)$ \\
& $\mathrm{p}=0.80$ & 0.66
\end{tabular}

Positive(+) beta coefficient indicates an increase in WOMAC pain.

*Model for each exposure and outcome: exposure +standard set of covariates (baseline age, sex, BMI, race, depressive symptoms and pain)+baseline BML score +baseline synovitis score.

†Values are unstandardised regression coefficients representing mean change in WOMAC knee pain over 24 or 36 months.

BML, bone marrow lesion; WOMAC, Western Ontario and McMaster Universities Osteoarthritis Index.

cartilage thickness gave a slightly stronger association with pain $(0.35,95 \%$ CI $0.20,0.51)$.

A subanalysis excluding valgus knees also did not appreciably change the results from the primary analysis in the whole sample. Secondary analyses using semiquantitative MOAKS measures of cartilage loss gave similar results to those using quantitative cartilage measures, including a weak association with change in WOMAC pain, mediated by change in synovitis score but not in BML score.

\section{DISCUSSION}

In this longitudinal study of individuals from the OAI, we found that cartilage thickness loss was associated with worsening knee pain after adjusting for coexistent BMLs and synovitis scores. While statistically significant, the relation between cartilage loss and worsening pain was, at best, modest with $0.1 \mathrm{~mm}$ of cartilage loss associated with $<1$ point worsening on a $0-20$ WOMAC pain scale. This suggests that it will be extremely hard to demonstrate that preventing cartilage loss reduces pain in a knee with OA. We also reported that the association of cartilage loss with pain was mediated, in part, by worsening synovitis, but not change in BMLs.

These results are concordant with other longitudinal studies of cartilage thickness loss and pain, ${ }^{613}$ although those studies did not quantify the relation of cartilage loss with pain nor examine synovitis and BMLs as additional sources of pain.

Our analysis used WOMAC pain as our pain outcome, but the impact of OA on patient outcomes is complex. WOMAC is a validated instrument that targets pain severity during specific activities likely to cause OA pain, but it is possible that the effects of cartilage loss would be captured better with an instrument that focused more on the quality or persistence of

Table 3 Mediation effects for outcome WOMAC pain over 24 months: association of change in cartilage thickness loss over 24 months, with change in WOMAC knee

\begin{tabular}{|c|c|c|}
\hline Exposure: cartilage thickness loss over 24 months & $\begin{array}{l}\text { Mean change in WOMAC knee pain over } 24 \\
\text { months* }\end{array}$ & $\begin{array}{l}\text { Mean change in WOMAC knee pain over } 24 \\
\text { months* }\end{array}$ \\
\hline \multicolumn{3}{|l|}{ Exposure: $0.1 \mathrm{~mm}$ cartilage thickness loss over 24 months } \\
\hline Controlled direct effect & $0.28(-1.68$ to 2.24$) \dagger$ & $0.33(-1.62$ to 2.30$) \dagger$ \\
\hline Indirect effect & $0.05(-1.91$ to 2.01$)$ & $0.01(-1.95$ to 1.97$)$ \\
\hline Total effect & $0.33(-1.63$ to 2.29$)$ & $0.34(-1.62$ to 2.30$)$ \\
\hline $\begin{array}{l}\text { Proportion } \\
\text { mediated }\end{array}$ & $14.11 \%(2.07 \%$ to $26.15 \%)$ & $2.77 \%(-1.71 \%$ to $7.25 \%)$ \\
\hline \multicolumn{3}{|l|}{ Exposure: $0.05 \mathrm{~mm}$ cartilage thickness loss over 24 months } \\
\hline Controlled direct effect & $0.14(-1.82$ to 2.10$)$ & $0.17(-1.79$ to 2.13$)$ \\
\hline Indirect effect & $0.02(-1.92$ to 1.98$)$ & $0.004(-1.96$ to 1.96$)$ \\
\hline Total effect & $0.16(-1.80$ to 2.12$)$ & $0.17(-1.79$ to 2.13$)$ \\
\hline $\begin{array}{l}\text { Proportion } \\
\text { mediated }\end{array}$ & $14.11 \%(2.07 \%$ to $26.15 \%)$ & $2.77 \%(-1.71 \%$ to $7.25 \%)$ \\
\hline
\end{tabular}

Positive(+) coefficient indicates an increase in the outcome WOMAC pain.

Models for mediation by change in synovitis score adjusted for standard covariates above +baseline BML score.

Models for mediation by change in BML score adjusted for standard covariates above +baseline synovitis score.

${ }^{*}$ All models adjusted for age, sex, BMI, race, depressive symptoms (CESD) and baseline WOMAC pain.

†Values are unstandardised regression coefficients representing mean change in WOMAC knee pain over 24 months.

BMI, body mass index; BML, bone marrow lesion; CESD, Center for Epidemiologic Studies Depression Scale; WOMAC, Western Ontario and McMaster Universities Osteoarthritis Index. 
Table 4 Mediation effects for outcome WOMAC pain over 36 months: association of change in cartilage thickness loss over 24 months, with change in WOMAC knee

\begin{tabular}{|c|c|c|}
\hline & $\begin{array}{l}\text { Mediator: synovitis score change over } 24 \text { months } \\
\text { Mean change in WOMAC knee pain over } 36 \text { months }\end{array}$ & $\begin{array}{l}\text { Mediator: BML score change over } 24 \text { months } \\
\text { Mean change in WOMAC knee pain over } 36 \text { months }\end{array}$ \\
\hline \multicolumn{3}{|c|}{ Exposure: $0.1 \mathrm{~mm}$ cartilage thickness loss over 24 months } \\
\hline Controlled direct effect & 0.16 (-1.80 to 2.12$)$ & $0.24(-1.73$ to 2.19$)$ \\
\hline Indirect effect & $0.04(-1.92$ to 2.00$)$ & $-0.003(-1.96$ to 1.96$)$ \\
\hline Total effect & 0.16 (-1.77 to 2.15$)$ & $0.23(-1.73$ to 2.19$)$ \\
\hline $\begin{array}{l}\text { Proportion } \\
\text { mediated }\end{array}$ & $19.78 \%(-1.65 \%$ to $41.21 \%)$ & Proportion too small to calculate- - not a mediator \\
\hline \multicolumn{3}{|c|}{ Exposure: $0.05 \mathrm{~mm}$ cartilage thickness loss over 24 months } \\
\hline Controlled direct effect & 0.08 (-1.88 to 2.04$)$ & $0.12(-1.84$ to 2.08$)$ \\
\hline Indirect effect & $0.02(-1.94$ to 1.98$)$ & $-0.002(-1.96$ to 1.96$)$ \\
\hline Total effect & 0.10 (-1.86 to 2.06$)$ & 0.12 (-1.84 to 2.08$)$ \\
\hline $\begin{array}{l}\text { Proportion } \\
\text { mediated }\end{array}$ & $19.78 \%(-1.65 \%$ to $41.21 \%)$ & Proportion too small to calculate- - not a mediator \\
\hline
\end{tabular}

All models adjusted for age, sex, BMI, race and depressive symptoms (CESD).

Positive(+) coefficient indicates an increase in the outcome WOMAC pain.

Models for mediation by change in synovitis score adjusted for standard covariates above +baseline BML score.

Models for mediation by change in BML score adjusted for standard covariates above +baseline synovitis score.

BMI, body mass index; BML, bone marrow lesion; CESD, Center for Epidemiologic Studies Depression Scale; WOMAC, Western Ontario and McMaster Universities Osteoarthritis Index.

pain. Cartilage loss might make pain more continuous and this persistence might be lessened by a chondroprotective agent. Or cartilage loss might affect physical function more than pain severity. Furthermore, cartilage loss was slow in this subgroup and it is conceivable that the association of cartilage loss with pain worsening would be stronger among knees with rapid progression. Lastly, while we did not find that most of the effect of cartilage loss on pain was mediated by synovitis or BMLs, there is considerable evidence that synovitis and BMLs are sources of pain in OA; it may be that this pain is not mostly triggered by cartilage loss.

The recent FDA guidance on OA states 'To accept structural endpoints as valid outcome measures for accelerated approval, there should be substantial confidence, ... That an effect on the candidate structural endpoint will reliably predict an effect on the clinical outcomes of interest.'. ${ }^{29}$ The clinical outcomes of interest are, in this case, patient pain or function. Our data suggest that these effects, using the WOMAC pain scale, are detectable but minimal.

A key strength of this analysis is the use of quantitative measures of cartilage in the medial joint, measured from MRI images. These measures should be sensitive to change in cartilage, although they may also include some measurement error. ${ }^{6}$ To get a sense of how much effect on cartilage may occur with drugs or biologics, we examined the trial of sprifermin ${ }^{14}$ which tested multiple dosing regimens against placebo on cartilage thickness over 2 years. While most doses had standardised response means (SRMs) of less than $1 / 2 \mathrm{SD}$, the most potent dose, $100 \mu \mathrm{g}$ every 6 months, had an SRM of 0.68 SDs over 2 years. This is a large treatment effect for OA therapeutics ${ }^{30}$ and for chondroprotection, by far, the largest effect seen. If a treatment protected against $0.1 \mathrm{~mm}$ of cartilage loss over 2 years versus placebo, it would be expected to generate a WOMAC pain difference of 0.32 on the $0-20$ scale. The minimal clinically important difference (MCID) of WOMAC pain varies but is generally $\geq 2,{ }^{31}$ and there has been little work estimating whether it is similar for worsening, but we strongly suspected that the average treated patient would not experience a pain effect approaching an MCID.

Our analysis has potential limitations. There may be residual confounding, possibly including other sources of pain change.
We also expected that regression to the mean for pain occurred in this cohort, particularly for measures of pain but that should have affected both those experiencing and those not experiencing cartilage loss. Individuals participate in studies when they are symptomatic (ie, in more pain), and over time as they improve, pain severity may lessen, regressing to their mean. This would also be true in clinical trials, where patients often seek treatment when they are in considerable pain. Also, there may also be selection bias due to the sampling strategy of the FNIH sample described earlier. Furthermore, the subjects in the FNIH sample are not likely to be generalisable to subjects entering trials in OA. On average, for example, they had less severe knee pain. Even so, we noted that a recent OA trial found no association of chondroprotection with effects on pain severity.

In conclusion, cartilage thickness loss is weakly associated with knee pain, and the relationship is so weak as to call into question attempts to develop treatments that target only chondroprotection. The association with pain appears to be partially mediated by change in synovitis and may be greater in those with no knee pain than in those with pre-existing pain.

Acknowledgements Financial support from NIH grants P30AR072571, T32 AR7598. Data provided from the FNIH OA Biomarkers Consortium Project are made possible through grants and direct or in-kind contributions by: AbbVie; Amgen; Arthritis Foundation; Artialis; Bioiberica; BioVendor; DePuy; Flexion Therapeutics; GSK; IBEX; IDS; Merck Serono; Quidel; Rottapharm | Madaus; Sanofi; Stryker; the Pivotal OAI MRI Analyses (POMA) study; NIH HHSN2682010000 21C and the Osteoarthritis Research Society International. There are no competing interests.

Contributors KB: Conception and design, drafting of article, performing analysis. MPL and SRJ: editing article, overseeing analysis. DF: Conception and design, drafting of article. All authors: final approval of manuscript.

Funding This study was funded by National Institutes of Health; grant number (P30 AR072571)

\section{Competing interests None declared.}

Patient and public involvement Patients and/or the public were not involved in the design, or conduct, or reporting, or dissemination plans of this research.

Patient consent for publication Not required.

Provenance and peer review Not commissioned; externally peer reviewed. 
Data availability statement Data are available in a public, open access repository. The data used for analyses in this paper are publicly available at https:// nda.nih.gov/oai.

ORCID iDs

S Reza Jafarzadeh http://orcid.org/0000-0002-1099-9175

David Felson http://orcid.org/0000-0002-2668-2447

\section{REFERENCES}

1 Felson DT. Developments in the clinical understanding of osteoarthritis. Arthritis Res Ther 2009; 11:203.

2 Felson DT, Chaisson CE, Hill CL, et al. The association of bone marrow lesions with pain in knee osteoarthritis. Ann Intern Med 2001:134:541-9.

3 Blaney Davidson EN, van Caam APM, Vitters EL, et al. TGF- $\beta$ is a potent inducer of Nerve Growth Factor in articular cartilage via the ALK5-Smad2/3 pathway. Potential role in OA related pain? Osteoarthritis Cartilage 2015;23:478-86.

4 Felson DT, Niu J, Neogi T, et al. Synovitis and the risk of knee osteoarthritis: the most study. Osteoarthritis Cartilage 2016;24:458-64.

5 Zhang Y, Nevitt M, Niu J, et al. Fluctuation of knee pain and changes in bone marrow lesions, effusions, and synovitis on magnetic resonance imaging. Arthritis Rheum 2011;63:691-9.

6 Wluka AE, Wolfe R, Stuckey $S$, et al. How does tibial cartilage volume relate to symptoms in subjects with knee osteoarthritis? Ann Rheum Dis 2004;63:264-8.

7 Cooper C, Snow S, McAlindon TE, et al. Risk factors for the incidence and progression of radiographic knee osteoarthritis. Arthritis Rheum 2000:43:995-1000.

8 Dieppe PA, Cushnaghan J, Shepstone L. The Bristol 'OA500' study: progression of osteoarthritis (OA) over 3 years and the relationship between clinical and radiographic changes at the knee joint. Osteoarthritis Cartilage 1997;5:87-97.

9 Ledingham J, Regan M, Jones A, et al. Factors affecting radiographic progression of knee osteoarthritis. Ann Rheum Dis 1995;54:53-8.

10 Massardo L, Watt I, Cushnaghan J, et al. Osteoarthritis of the knee joint: an eight year prospective study. Ann Rheum Dis 1989;48:893-7.

11 Baum T, Joseph GB, Arulanandan A, et al. Association of magnetic resonance imagingbased knee cartilage $\mathrm{T} 2$ measurements and focal knee lesions with knee pain: data from the osteoarthritis initiative. Arthritis Care Res 2012;64:248-55.

12 Saunders J, Ding C, Cicuttini F, et al. Radiographic osteoarthritis and pain are independent predictors of knee cartilage loss: a prospective study. Intern Med J 2012:42:274-80.

13 Eckstein F, Collins J, Nevitt M, et al. Cartilage thickness change as an imaging biomarker of knee osteoarthritis progression - data from the FNIH oa biomarkers Consortium. Arthritis Rheumatol 2015;67:3184.

14 Hochberg MC, Guermazi A, Guehring H, et al. Effect of intra-articular Sprifermin vs placebo on femorotibial joint cartilage thickness in patients with osteoarthritis: the forward randomized clinical trial. JAMA 2019:322:1360-70.
15 Lester G. Clinical research in OA--the NIH Osteoarthritis Initiative. J Musculoskelet Neuronal Interact 2008:8:313-4.

16 Hunter DJ, Nevitt M, Losina E, et al. Biomarkers for osteoarthritis: current position and steps towards further validation. Best Pract Res Clin Rheumatol 2014:28:61-71.

17 Eckstein F, Maschek S, Wirth W, et al. One year change of knee cartilage morphology in the first release of participants from the osteoarthritis initiative progression subcohort: association with sex, body mass index, symptoms and radiographic osteoarthritis status. Ann Rheum Dis 2009:68:674-9.

18 Wirth W, Hellio Le Graverand M-P, Wyman BT, et al. Regional analysis of femorotibial cartilage loss in a subsample from the osteoarthritis initiative progression subcohort. Osteoarthritis Cartilage 2009;17:291-7.

19 Hunter DJ, Guermazi A, Lo GH, et al. Evolution of semi-quantitative whole joint assessment of knee oa: MOAKS (MRI osteoarthritis knee score). Osteoarthritis Cartilage 2011;19:990-1002.

20 McAlindon TE, LaValley MP, Harvey WF, et al. Effect of intra-articular triamcinolone vs saline on knee cartilage volume and pain in patients with knee osteoarthritis: a randomized clinical trial. JAMA 2017:317:1967-75.

21 Bellamy N, Buchanan WW, Goldsmith CH, et al. Validation study of WOMAC: a health status instrument for measuring clinically important patient relevant outcomes to antirheumatic drug therapy in patients with osteoarthritis of the hip or knee. $J$ Rheumatol 1988;15:1833-40.

22 Bellamy N. WOMAC osteoarthritis index. A user's guide. London, Ontario, Canada: University of Western Ontario, 1995.

23 Radloff LS. The CES-D scale: a self-report depression scale for research in the general population. Appl Psychol Meas 1977;1:385-401.

24 Valeri L, Vanderweele TJ. Mediation analysis allowing for exposure-mediator interactions and causal interpretation: theoretical assumptions and implementation with SAS and SPSS macros. Psychol Methods 2013:18:137-50.

25 Hertzmark E, Pazaris M, Spiegelman D. The SAS mediate macro. Boston: Harvard TH Chan School of Public Health, 2012

26 Nevo D, Liao X, Spiegelman D. Estimation and inference for the mediation proportion. Int J Biostat 2017;13. doi:10.1515/ijb-2017-0006. [Epub ahead of print: 20 Sep 2017].

27 Lin DY, Fleming TR, De Gruttola V. Estimating the proportion of treatment effect explained by a surrogate marker. Stat Med 1997;16:1515-27.

28 VanderWeele TJ. Mediation analysis: a practitioner's guide. Annu Rev Public Health 2016:37:17-32

29 FDA. Osteoarthritis: structural endpoints for the development of drugs, devices, and biological products for treatment guidance for industry, 2018. Available: http://www fda gov/downloads/Drugs/GuidanceCmplianceRegulatorylnformation/Guidances/ ucm071577 pdf

30 Felson DT, Neogi T. Emerging treatment models in rheumatology: challenges for osteoarthritis trials. Arthritis Rheumatol 2018;70:1175-81.

31 Olsen MF, Bjerre E, Hansen MD, et al. Minimum clinically important differences in chronic pain vary considerably by baseline pain and methodological factors: systematic review of empirical studies. J Clin Epidemiol 2018;101:e2:87-106. 